\title{
Remotely Controllable Wireless Road Stud Network
}

\author{
J.H. Le Roux, A. Barnard and M.J. Booysen \\ Department of Electrical and Electronic Engineering, Stellenbosch University \\ Stellenbosch, South Africa. Email: 15661563@sun.ac.za
}

\begin{abstract}
Developing countries face many infrastructure related challenges, one of them being road safety. This paper proposes a novel system introducing a network of wirelessly connected road studs that are remotely controllable from an online platform. In this proof-of-concept demonstrator, a smallscale set-up was designed and constructed to assist in the analysis of the requirements for such a system, which will form part of a greater ITS system. The system consists of three functional parts, namely the online SMART platform which is used to transmit commands to the road studs in the field via a GSM connection; the coordinator unit, which receives the commands through a GSM modem from the platform and then transmits the relevant data to the road studs through an IEEE 802.15.4 based wireless network; the road stud units, which receives commands from the coordinator and reconfigures its settings in terms of colour, brightness and flashing frequency accordingly. In this paper we review the design process and highlight some key results from the constructed model.
\end{abstract}

\section{INTRODUCTION}

Road safety is a critical challenge faced by all countries, especially developing countries. There is an abundance of possible road safety measures which can be implemented, but each has its own limitations in terms of cost, functionality and complexity. In the context of road safety in developing countries, scenarios exist where there is little or no infrastructure to support road safety, especially in rural areas. While the availability of electricity is a basic necessity for many Intelligent Transportation System (ITS) extensions in the developed world, its glaring absence in many developing areas limits many of the opportunities to improve road safety. In developing countries, there is a need for a road safety solution that is flexible and easy to implement in terms of cost and complexity. Moreover, its dependence on local infrastructure for electricity or other road safety mechanisms must be minimized.

The traditional reflective cat's eye has been in operation for decades and is used to visually delineate lanes at night, but advances in electronics have introduced the creation of solar powered Light Emitting Diode (LED) road studs. Solar road studs make use of LEDs to provide illumination, which are visible from up to 900 meters. This is a considerable improvement when compared to a reflective cat's eye which is only visible from up to $90 \mathrm{~m}$ [1].

Astucia, a leading road stud manufacturer, claims that its solar studs, which have been operationalised in more than 300 locations, have reduced road casualties by up to $72 \%$ on average [1]. Further statistics confirm a $72 \%$ reduction in night time accidents in South Wales. In Norfolk (UK) there was an overall accident frequency reduction from 7.3 accidents per year to 2.3 , with the severity ratio reducing from $36 \%$ to zero. Seven months prior to the installation of road studs on the R66 in South Africa (between Ulundi and Melmoth) 88 serious night time accidents, which claimed 27 lives, were recorded. There were no recorded night-time accidents on this stretch of road for 12 months following the installation of solar road studs [2]. It is clear that solar road studs have a proven record of significantly reducing road accidents.

\section{RELATED WORK}

Various road stud systems have been proposed to improve road safety. Some of the popular approaches, and the features they support, are listed in Table I.

There are a variety of road stud units commercially available, but these fulfil only the basic requirements in terms of LED illumination. Astucia appears to be leading the commercial market in road studs. It provides solar powered as well as hard wired solutions. The solar powered units provide battery operation, solar recharge capabilities and easy installation, but it lacks in terms of reconfigurability after installation [4]. The hard-wired solutions solve the lack of reconfigurability of the solar road studs, but at an increased cost and loss of easy installation [3]. The hardwired units have the ability to be remotely controlled, but the range and extent of control is unclear.

A promising project named Road Nail aims to bridge the gap in functionality between the solar road studs and hard wired studs [5]. Road Nail is a road stud system that is LED illuminated with two colours (red and white), with wireless communication functionality and is battery operated with solar recharging. The network has a cellular base station which can transmit information to and from a monitoring center. This will be used for infrastructure-to-vehicle (I2V) and vehicle-to-infrastructure (V2I) communication, but the exact purpose and goal of these systems are unclear. Road Nail's primary focus is on dynamic network topology discovery and vehicle detection. These elements are used to detect a vehicle and propagate a message through the network to instruct the studs to illuminate ahead of the vehicle.

The aforementioned aspects are not of significant importance to developing countries and vehicles in such countries will rarely have the ability to integrate with $\mathrm{I} 2 \mathrm{~V}$ and V2I systems. There is no indication of remote control capabilities, despite the inclusion of a cellular connection to the network and remote control would be limited since the device only supports two colours. 
TABLE I

SUMMARY OF PROPOSED ROAD STUD SOLUTIONS.

\begin{tabular}{|c|c|c|c|c|c|c|}
\hline System & LED Illumination & 3 Colours & Solar Powered & Wireless Functionality & Wireless Control & Ref. \\
\hline \hline Hard Wired Studs & $\sqrt{ }$ & $\sqrt{ }$ & - & - & - & {$[3]$} \\
\hline Solar Road Studs & $\sqrt{ }$ & - & $\sqrt{ }$ & - & - & {$[4]$} \\
\hline Road Nail & $\sqrt{ }$ & - & $\sqrt{ }$ & $\sqrt{ }$ & - & {$[5]$} \\
\hline Our Proposal & $\sqrt{ }$ & $\sqrt{ }$ & $\sqrt{ }$ & $\sqrt{ }$ & $\sqrt{ }$ & \\
\hline
\end{tabular}

Traffic management models [6] and vehicle detection sensors [7][8] have been developed by various institutions. The last mentioned projects describe the design and construction of road-stud-like devices that act solely as wireless sensors without any form of illumination.

\section{A. Contribution}

Although advances have been made to road stud technology, there is a lack of remote configurable studs which have the requirements of a developing country as its focus. An ITS solution aimed at developing countries must be easily deployable, highly configurable, easily controllable, cost effective and not be dependent on local infrastructure. This project aims to further improve on existing solar powered road studs by adding additional flexibility and functionality with remote configurabilty through an internet based platform and interstud wireless communication. This solution will primarily provide improvements to road safety, and secondly traffic management.

\section{SYSTEM DESIGN}

This section provides an overview of the involved networks as well as the system and hardware design.

\section{A. System Overview}

A diagrammatic representation of the system is shown in Fig. 1. The entire system relies on two networks, the coordinator network and the road stud network. The coordinator network is controlled from the SMART online platform and the road stud network will be managed by a coordinator node.

1) Internet Segment: The internet segment consists of a user interface, or dashboard, as well as a server and database segment. This entire segment is known as the SMART platform which is provided by Trinity Telecommunications [9]. The dashboard is used to configure the settings of any road stud network remotely. It is designed to provide a convenient and innovative solution for configuring the road studs. Access to the dashboard is achieved through the Internet, which allows multiple institutions to configure the network from any remote location.

2) Cellular Connection: The cellular connection to the studs in the field is facilitated by a Global System for Mobile Communications (GSM) modem which is connected to the coordinator unit situated adjacent to the road. This allows the deployment of road studs anywhere with cellular coverage, which is the case for almost all roads in developing countries.

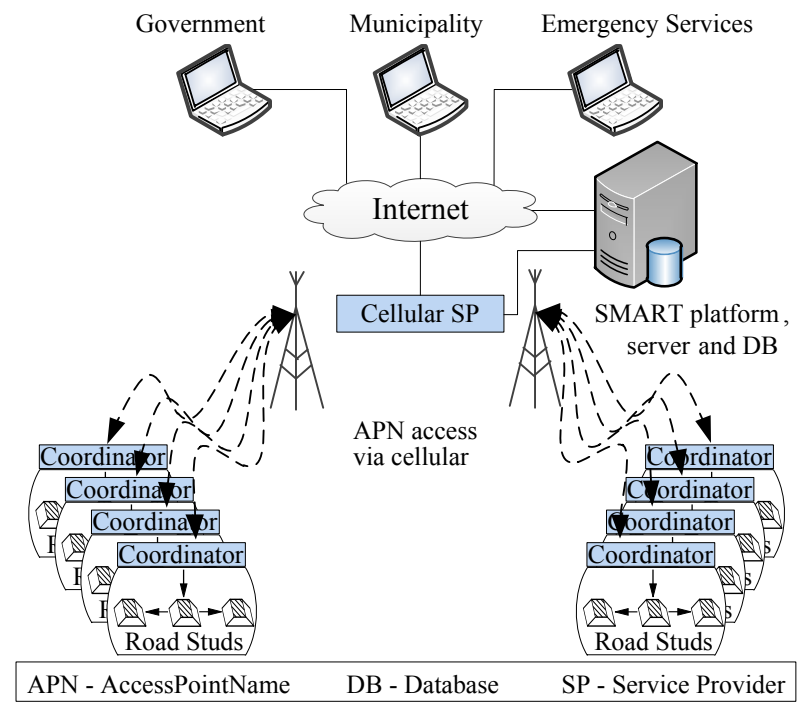

Fig. 1. System layout

The GSM connection for this system can be considered as transparent to the user, because it is entirely managed through the SMART platform. Where there is no cellular coverage, the studs can still be deployed with a pre-configured setting, but the ability to modify the configuration remotely will be limited.

3) Coordinator: The coordinator is a roadside base station that controls the road studs and communicate with the online platform via a GSM modem. It is physically separated from the road stud units since it is larger and requires more power to support the GSM modem. It will not be possible to fit the required hardware into a road stud housing. The coordinator acts as the leading node in the wireless road stud network. It transmits commands that it receives from the SMART platform to the road studs.

4) Road Stud: The road studs are bi-directional, LED illuminated units which are deployed onto the road surface to fulfil the role of the traditional cat's eye. Each stud has three colours in each direction (red, yellow and white) as defined by the South African Development Community Road Traffic Signs Manual (SADCRTSM) [10]. The studs are battery operated and will rely on a basic solar powered recharge mechanism to recharge during daylight conditions. Configuration instructions originate at the coordinator and are transmitted to the road studs. 


\section{B. SMART Platform}

The SMART platform is used to manage the road stud networks, since it allows for the convenient visualisation and customisation of the road network. It provides a dashboard (user interface) which can be used to adjust the road stud configurations. Fig. 2 provides a concept dashboard design that could be used for such a system. The dashboard provides

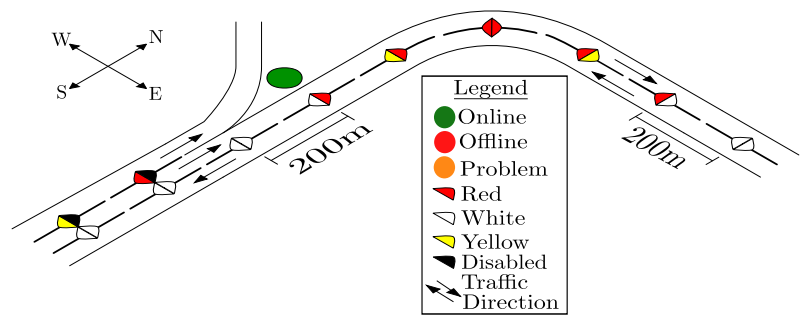

Fig. 2. Concept dashboard for online platform.

the functionality that is required to control a road stud network. The coordinator is represented by a large circle and the road studs in each segment by two half circles, one half for each direction. The road in this example is divided into $200 \mathrm{~m}$ segments with 10 road studs per segment. The road segment parameters can be scaled according to road topology. The configuration of a segment can be adjusted by selecting and modifying the appropriate half circle. Different pre-sets for the specified coordinator can be stored for easy assignment depending on the road conditions.

\section{Hardware Design}

1) Microprocessor and Radio: The Texas Instruments CC2530 System-on-Chip (SoC) was identified as the microprocessor for the experimental board. It was specifically selected as it contains an integrated $2.4 \mathrm{GHz}$ radio transceiver based on the IEEE 802.15.4 standard, which is a low-cost and low power protocol with a raw data rate of $250 \mathrm{kbps}$ on the worldwide available $2.4 \mathrm{GHz}$ channel [11]. The processor reduces complexity as it allows for a small physical footprint as very little peripheral components are required for operation.

2) Power Supply: The power supply consists of a basic solar rechargeable battery circuit which supplies energy to the device. The battery supplies two voltage boost-converters, with efficiencies up to $96 \%$, which are used to boost the lower battery voltage to the required levels of 3.3 and $5 \mathrm{~V}$. The $3.3 \mathrm{~V}$ is used to power the logical circuits and the $5 \mathrm{~V}$ is used to power the LEDs, since the LEDs require a higher voltage to operate. Normal AAA rechargeable batteries are used for energy storage. Super capacitors where considered as possible energy storage devices, but it did not conclude to an effective solution when compared to chemical batteries in terms of cost and availability.

3) Antenna Design: The antenna design for the project is based on a Texas Instruments design note. This PCB-based antenna has a small physical footprint, a negligible cost and a reported line of sight range of $240 \mathrm{~m}$ [12]. This range is more than adequate as the maximum road stud spacing in
South Africa is only 24 meters due to the regulations set out in the SADCRTSM.

4) Modem: The modem utilised for this project is a Sierra Wireless AirLink GL6100 modem and operates on custom firmware designed by Trinity. It facilitates a GSM connection to the SMART platform and provides a local communication interface on a serial RS232 connection through a DB9 header at a data rate of 115200 bps.

5) Illumination: The road stud's illumination relies on high powered LEDs. One stud contains six LEDs, two sets of red, white and yellow. One set for the front and one for the back. This allows for the bi-directional functionality of the unit. The LEDs are controlled by the microprocessor and a LED driving circuit. The LED driving circuits were designed to be adjustable to reduce the power consumption, since the illumination consumes the bulk of the power on the device.

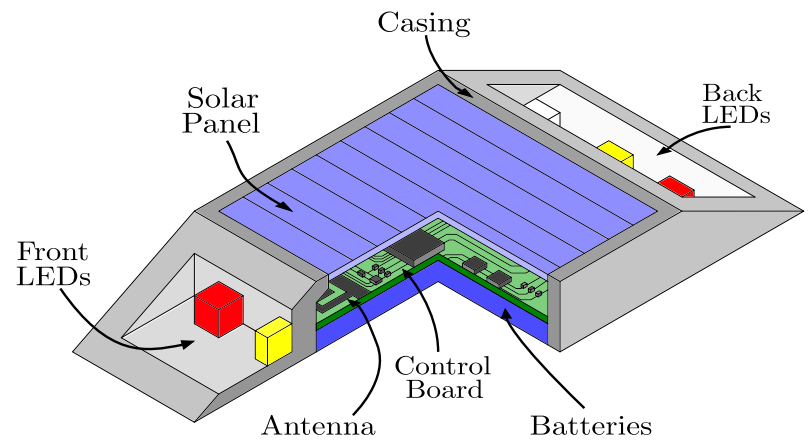

Fig. 3. Cut-away concept casing design for the road stud unit.

6) Road Stud Housing: A concept casing design of the road stud unit is illustrated in Fig. 3. This concept housing has a low profile and is installed on the road surface which allows for easy deployment and replacement in the event of damage.

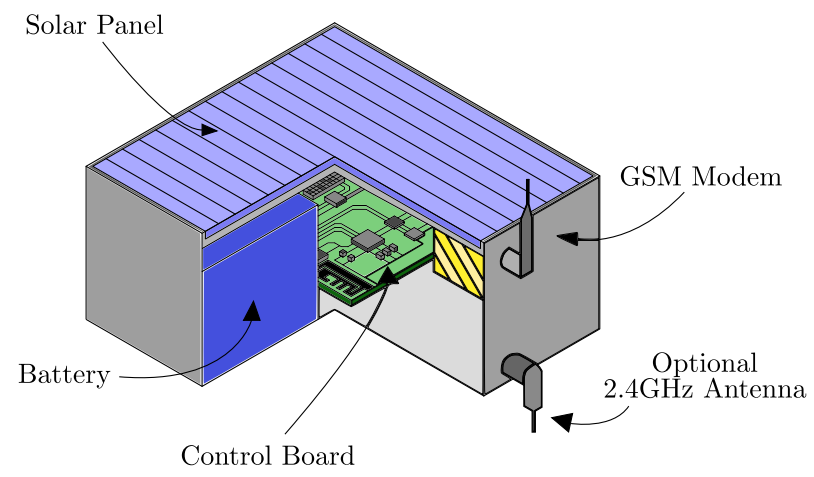

Fig. 4. Cut-away concept casing design for the pole-mounted coordinator unit.

7) Coordinator Housing: Since the coordinator unit will be deployed adjacent to the road, it will require a rugged/weatherproof housing. It should be mounted on a pole for increased security, improved wireless range and GSM connectivity. Fig. 4 illustrates a concept design of a coordinator housing. The housing will house all the required components while the solar panels will be mounted on top of the casing. 


\section{Road Stud Network}

The desired road stud network will utilise a data hopping network protocol as illustrated in Fig. 5. If the network had a star topology, all the road studs would need to be in direct communication range with the coordinator. This would severely limit the network range as the antenna can only function up to 240 meters under good conditions. The data hopping protocol primarily extends the effective communication range of the network and, in effect, the total amount of nodes.

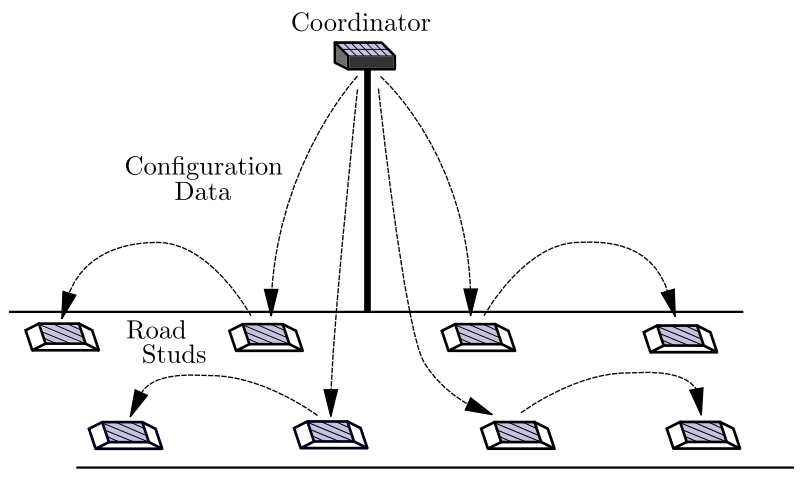

Fig. 5. Data hopping in the road stud network.

A 16-bit addressing protocol will enable up to 65534 nodes under control of a single coordinator. The network protocol transmits 8-bit commands to the road studs, which allows up to 256 unique system commands per stud. The network will not require fast transmission rates as the data packages are small. A few seconds delay in the communication system will have little to no influence on the functionality as the system is not highly time critical.

\section{Cost Estimate}

TABLE II

Cost Estimate Of Components

\begin{tabular}{|c|l|}
\hline Item & Cost \\
\hline \hline CC2530 Microprocessor & 6.5 EUR \\
\hline Power Circuits & 4.3 EUR \\
\hline Batteries & 3.6 EUR \\
\hline Solar Panel & 7.2 EUR \\
\hline LEDs & 4.3 EUR \\
\hline LED Driving Circuits & 1.4 EUR \\
\hline Modem Communication Circuits & 6.1 EUR \\
\hline Peripheral Circuits & 3.6 EUR \\
\hline PCB Manufacturing & 6.0 EUR \\
\hline Assembly & 1.5 EUR \\
\hline
\end{tabular}

A preliminary cost estimate was done and the estimates of individual components can be viewed in Table II. A coordinator unit will cost in the range of 55.00 EUR per unit (excluding the modem and housing). A road stud unit will cost an estimated 38.00 EUR per unit (excluding housing).
1600 Road stud units will cost in the region of 64000 EUR, which is enough studs to cover a $10 \mathrm{~km}$ stretch of highway with two lanes and two emergency lanes with 24 meter road stud spacing.

\section{EXPERIMENTAL BOARD AND DISCUSSION}

Fig. 6 illustrates the experimental hardware platform. A modular hardware platform was designed where components can be enabled or disabled to configure the functionality of the device. The configuration determines if the unit acts as a coordinator or a road stud. The experimental PCB was developed on a two layer board and the platform was designed to be large, as it allows for easy testing.

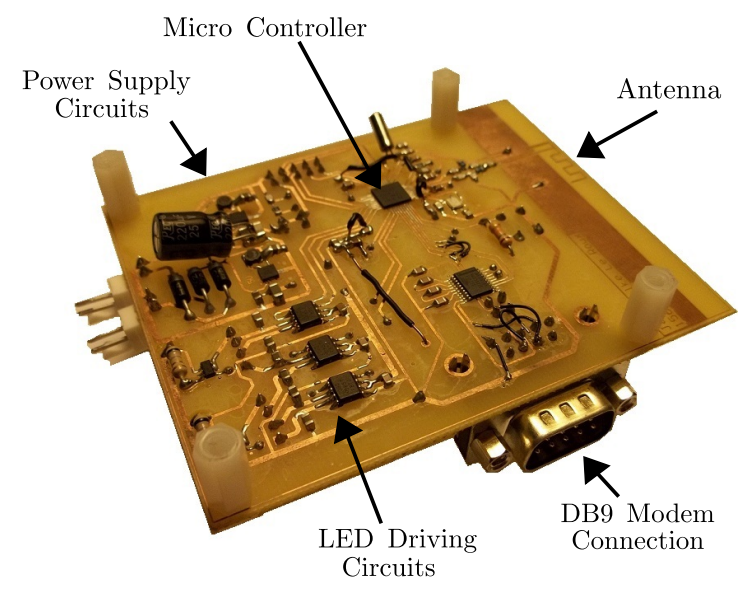

Fig. 6. Experimental road stud/coordinator unit

The experimental network protocol relies on a basic star topology where data messages were passed from the coordinator to each individual road stud. This protocol supports basic networking functions and it was used to demonstrate the network functionality and range. The experimental device was tested up to a wireless communication range of 30 meters in a noisy electromagnetic environment. With the test range of 30 meters, the device would be able to function well within its application, as the studs will not be placed further than 24 meters apart in South Africa. The size of the antenna is comparable to the footprint of the standard DB9 connector and is shown in Fig. 6. An online dashboard was developed, which provides some basic functionality to configure the road stud unit.

The entire system was successfully tested. Configurations were changed on the online dashboard and the messages propagated through the entire system up to the road stud units.

In developing countries it often occurs that roads with low traffic conditions suddenly become high traffic areas due to economic expansion, especially in the proximity of urban areas. This is where the proposed solar powered road stud system will be of most value, as it can be deployed on existing roads, and it is independent of local power sources. The added traffic management capability through dynamic lane configuration could possibly increase traffic efficiency 
under high load conditions. Dynamic lane switching can allow effortless traffic management as well as improve safety in emergency situations. For example if there is an accident or road obstruction, then an entire lane can be de-allocated for a certain section.

The system can be highly effective at increasing road safety, but additions can be made in the future to expand its functionality. The coordinator unit as well as the road studs can house additional sensors to allow more data to be captured since the developed hardware platform does allow for the implementation of additional sensors. The SMART platform provides extensive data processing and visualisation tools. The additional sensors coupled with the tools provided by the SMART platform would allow for the capture of statistics to analyse traffic patterns and improve traffic management even further. Coordinators could possibly house vehicle counting and weather sensing equipment. Road studs can house moisture and vehicle detection or counting sensors as some experimentation has been done with these types of sensors [7][8].

The South Africa Road Accident Fund reported that in 2012 the average claim for an accident was 4384 EUR [13]. Using this average and applying it to the R66 road mentioned in Section I, an estimated damage cost can be calculated. The amount claimed for the 88 accidents would total 385 848 EUR, where the estimated total cost of installing the proposed road studs would be approximately 210000 EUR. This is a reduction of $45 \%$. The proposed road stud clearly offers a financial advantage, and will most possibly be more efficient and save lives.

\section{CONCLUSION}

This paper described the design of a wireless controllable solar powered road stud network system which relies on a GSM connection as well as a local $2.4 \mathrm{GHz}$ wireless connection. The system allows for dynamic lane configuration through an online dashboard which can be accessed by multiple institutions. The system should provide notable improvements to road safety and traffic management. It is particularly aimed at developing countries, as it can be deployed on existing roads and it is not reliant on local energy sources.

Future work will include: power consumption analysis, improvements to the network protocol, hardware refinements and housing development.

\section{REFERENCES}

[1] G. Muspratt, "Five ways to make night-time driving safer with Astucia Active Road Studs," Astucia, White Paper, December 2012.

[2] K. Donegan, "Internally Illuminated Road Studs," Intergrated Traffic Solution, Tech. Rep., 2011.

[3] Astucia. (2010) Intelligent Road Studs - Hardwired Systems. Clearview Traffic Group. [Online]. Available: http://www.civic.ie/Astucia\%20Hardwired\%20Systems.pdf

[4] Astucia. (2013) Astucia road studs. [Online]. Available: http://www.clearviewtraffic.com/astucia/

[5] D. Samardžija, N. Teslić, B. M. Todorović, E. Kovač, D. Isailović, and B. Miladinović, "Road Nail: Experimental Solar Powered Intelligent Road Marking System," Journal of Electrical Engineering, vol. 63, no. 2, pp. 65-74, 2012.

[6] J. Xu and Z. Huang, "An Intelligent model for Urban Demandresponsive Transport System Control," Journal of Software, vol. 4, no. 7, pp. 766 - 776, September 2009.

[7] A. N. Knaian, "A Wireless Sensor Network for Smart Road and Intelligent Transportation Systems," Master's thesis, Masssachusetts Institute of Technology, June 2000.

[8] F. Ahdi, M. K. Khandani, M. Hamedi, and A. Haghani, "Traffic data collection and anonymous vehicle detection using wireles sensor networks," University Of Maryland, Research Report, May 2012.

[9] Trinity Telecommunications. SMART Platform. [Online]. Available: http://www.trintel.co.za/

[10] D. Bain, South African Dept. of Transport, South African Roads Board, De Leuw Cather, SADC Working Group for the Harmonization of Road Traffic, and COLTO Road Traffic Signs Technical Committee South Africa, South Afican Development Community Road Traffic Signs Manual. South African Deartment Of Transport, November 1997, vol. 1.

[11] B. Latré, P. D. Mill, I. Moerman, N. V. Dierdonck, B. Dhoedt, and P. Demeester, "Maximum Throughput and Minimum Delay in IEEE 802.15.4," Department of Information Technology, Gent University and Ubiwave NV, Tech. Rep., 2005.

[12] A. Anderson, "Small Size 2.4GHz PCB Antenna," Texas Instruments, Application Note AN043, 2008.

[13] South African Road Accident Fund. (2012, March) Intergrated Annual Report. [Online]. Available: http://www.raf.co.za/MediaCenter/Documents/RAF\%20Annual\%20Report\%202012.pdf 